Advances in Dynamical Systems and Applications (ADSA).

ISSN 0973-5321, Volume 16, Number 1, (2021) pp. 75-89

(C) Research India Publications

https://dx.doi.org/10.37622/ADSA/16.1.2021.75-89

\title{
Ulam-type Stability for a Boundary Value Problem of Implicit Fractional-orders Differential Equation
}

\author{
A. M. A. El-Sayed ${ }^{1}$, Sh. M Al Issa ${ }^{2}$, and M. Elmiari ${ }^{2}$ \\ ${ }^{1}$ Faculty of Science, Alexandria University, Alexandria, Egypt. \\ ${ }^{2}$ Faculty of Science, Lebanese International University, Saida, Lebanon
}

\begin{abstract}
We discuss sufficient conditions for the existence of solutions for a boundary value problem of implicit fractional-orders differential equation. Some types of Ulam stability for our problem will bee establish.

Keywords and Phrases: Implicit differential-orders differential equation, Caputo derivative, existence results, boundary value problems, Green's function, Ulam stability.
\end{abstract}

MSC 2010: Primary 26A33; Secondary 34K45, 47G10.

\section{INTRODUCTION}

Recently, some mathematicians considered boundary value problems for fractional-orders differential equations (see [1]-[5], [7]-[22] and references therein). Let $1<\beta<\alpha \leq 2$. Here, we establish existence, Uniqueness and stability results for the boundary value problem of the implicit fractional-orders differential problem (IFDP):

$$
\begin{gathered}
{ }^{c} D^{\alpha} y(t)=f\left(t, y(t),{ }^{c} D^{\beta} y(t), \int_{0}^{t} k(t, s)^{c} D^{\alpha} y(s) d s\right), t \in I=[0 ; T], \\
y(0)=y_{\circ}, y(T)=y_{T} .
\end{gathered}
$$

The paper is organized as follows: Section 2, we present our main result by using Schauder's fixed point theorem. Furthermore, we prove the stability of solution of our problem. In Section 3, we give examples to demonstrate the application of our main results. Finally, we present conclusion in Section 4. 


\section{EXISTENCE OF SOLUTIONS}

Consider the problem (1)-(2) under the following assumptions

$\left(H_{1}\right) f: I \times R^{3} \rightarrow R$ is continuous and there exists $\psi \in C\left(I, R_{+}\right)$, with norm $\|\psi\|$, such that:

$$
\left|f\left(t, u_{1}, u_{2}, u_{3}\right)-f\left(t, v_{1}, v_{2}, v_{3}\right)\right| \leq \psi(t)\left(\left|u_{1}-v_{1}\right|+\left|u_{2}-v_{2}\right|+\left|u_{3}-v_{3}\right|\right)
$$

$\forall t \in I, u_{i}, v_{i} \in R,(i=1,2,3)$.

$\left(H_{2}\right) k(t, s)$ is continuous for all $(t, s) \in I \times I$, and there is a positive constant $K$ such that

$$
\max _{t, s \in[0, T]}|k(t, s)|=K
$$

\section{Remark:}

From assumption $\left(H_{1}\right)$, we have

$$
\begin{aligned}
\left|f\left(t, u_{1}, u_{2}, u_{3}\right)\right|-|f(t, 0,0,0)| & \leq\left|f\left(t, u_{1}, u_{2}, u_{3}\right)-f(t, 0,0,0)\right| \\
& \leq \psi(t)\left(\left|u_{1}\right|+\left|u_{2}\right|+\left|u_{3}\right|\right),
\end{aligned}
$$

then

$$
\left|f\left(t, u_{1}, u_{2}, u_{3}\right)\right| \leq \psi(t)\left(\left|u_{1}\right|+\left|u_{2}\right|+\left|u_{3}\right|\right)+|f(t, 0,0,0)|,
$$

and

$$
\left|f\left(t, u_{1}, u_{2}, u_{3}\right)\right| \leq\|\psi\|\left(\left|u_{1}\right|+\left|u_{2}\right|+\left|u_{3}\right|\right)+F, \text { where } F=\sup _{t \in I}|f(t, 0,0,0)| .
$$

Lemma 1. If the solution of IFDP (1)-(2) exist, it can be represented by the integral equation

$$
y(t)=h(t)+\int_{0}^{T} G(t, s) u(s) d s
$$

where $u$ is the solution of the functional integral equation

$$
u(t)=f\left(t, h(t)+\int_{0}^{T} G(t, s) u(s) d s, I^{\alpha-\beta} u(t), \int_{0}^{t} k(t, s) u(s) d s\right),
$$

$G(t, s)$ is the Green's function defined by

$$
G(t, s)= \begin{cases}\frac{(t-s)^{\alpha-1}}{\Gamma(\alpha)}-\frac{t(T-s)^{\alpha-1}}{T \Gamma(\alpha)} & 0 \leq s \leq t \leq T, \\ \frac{-t(T-s)^{\alpha-1}}{T \Gamma(\alpha)} & 0 \leq t \leq s \leq T\end{cases}
$$


with

$$
G_{0}:=\max \{|G(t, s)|,(t, s) \in I \times I\},
$$

and

$$
h(t)=y_{\circ}+\frac{\left(y_{T}-y_{\circ}\right) t}{T} .
$$

Proof. It is not difficult (see [21]) to verify that ${ }^{c} D^{\beta} y(t)=I^{\alpha-\beta c} D^{\alpha} y(t)$ for $t \in I$. If $y$ is a solution of equation (1), then

$$
{ }^{c} D^{\alpha} y(t)=f\left(t, y(t), I^{\alpha-\beta c} D^{\alpha} y(t), \int_{0}^{t} k(t, s){ }^{c} D^{\alpha} y(s) d s\right), \quad t \in J=:(0 ; T] .
$$

Let ${ }^{c} D^{\alpha} y(t)=u(t)$ in equation (1), then

$$
u(t)=f\left(t, y(t), I^{\alpha-\beta} u(t), \int_{0}^{t} k(t, s) u(s) d s\right)
$$

and

$$
y(t)=c_{\circ}+c_{1} t+\frac{1}{\Gamma(\alpha)} \int_{0}^{t}(t-s)^{\alpha-1} u(s) d s
$$

from which can get $c_{o}=y_{o}$ and

$$
c_{1}=-\frac{1}{T \Gamma(\alpha)} \int_{0}^{T}(T-s)^{\alpha-1} u(s) d s+\frac{\left(y_{T}-y_{\circ}\right)}{T} .
$$

Then the solution of (1)-(2) is given by

$$
\begin{aligned}
y(t) & =\frac{1}{\Gamma(\alpha)} \int_{0}^{t}(t-s)^{\alpha-1} u(s) d s-\frac{t}{T \Gamma(\alpha)} \int_{0}^{T}(T-s)^{\alpha-1} u(s) d s+\left(1-\frac{t}{T}\right) y_{\circ}+\frac{t}{T} y_{1} \\
& =\frac{1}{\Gamma(\alpha)}\left[\int_{0}^{t}\left[(t-s)^{\alpha-1}-\frac{t}{T}(T-s)^{\alpha-1}\right] u(s) d s-\frac{t}{T} \int_{t}^{T}(T-s)^{\alpha-1} u(s) d s\right] \\
& +\left(1-\frac{t}{T}\right) y_{\circ}+\frac{t}{T} y_{1} .
\end{aligned}
$$

As a result, we have equation (3) and (4).

Definition 1. By a mild solution of IFDP (1)-(2), we mean a function $u \in C(I, R)$ satisfying integral equation (4).

Our first existence result is based on Schauder's fixed point theorem.

Theorem 1. Let $\left(H_{1}\right)-\left(H_{2}\right)$ be hold. If

$$
\frac{\|\psi\|}{1-\aleph}<1, \quad \aleph=\frac{T^{\alpha-\beta}\|\psi\|}{\Gamma(\alpha-\beta+1)}+\|\psi\| K T .
$$

Then the IFDP (1)-(2) has at least one mild solution on I. 
Proof. Transform the IFDP (1)-(2) into a fixed point problem. Define the operator $A: C(I, R) \rightarrow C(I, R)$ by:

$$
A y(t)=h(t)+\int_{0}^{T} G(t, s) v(s) d s,
$$

where $v \in C(I, R)$ satisfies the implicit functional equation

$$
v(t)=f\left(t, y(t), I^{\alpha-\beta} v(t), \int_{0}^{t} k(t, s) v(s) d s\right) .
$$

and

with $G$ are the functions defined by (5).

$$
h(t)=y_{\circ}+\frac{\left(y_{T}-y_{\circ}\right) t}{T}
$$

Define the ball

$$
B_{\varrho}=\{y \in C(I, R):\|y\| \leq \varrho\}, \varrho \geq \frac{\left|y_{T}\right|+\frac{G_{\circ} T F}{1-\aleph}}{1-\frac{G_{\circ} T\|\psi\|}{1-\aleph}} .
$$

It is clear that the set $B_{\varrho}$ is nonempty, bounded, closed and convex. We demonstrate that the operator $A$ defined by (7) meets the hypothesis of the fixed point theorem of Schauder. The proof will be presented in several steps.

Step.1 : The operator $A$ is continuous.

Consider a sequence $\left\{x_{n}\right\} \subset B_{\varrho}$ such that $x_{n} \rightarrow x$ in $B_{\varrho}$. To show that $A$ is continuous, we have to prove that

$$
\left\|A x_{n}-A x\right\| \rightarrow 0 \text { as } n \rightarrow \infty .
$$

For this, we have

$$
\left|A x_{n}(t)-A x(t)\right| \leq \int_{0}^{T}|G(t, s)|\left|u_{n}(s)-u(s)\right| d s
$$

where $u_{n}, u \in C(I, R)$, such that

$$
\begin{aligned}
u_{n}(t) & =f\left(t, x_{n}(t), I^{\alpha-\beta} u_{n}(t), \int_{0}^{t} k(t, s) u_{n}(s) d s\right), \\
u(t) & =f\left(t, x(t), I^{\alpha-\beta} u(t), \int_{0}^{t} k(t, s) u(s) d s\right)
\end{aligned}
$$

and by $\left(H_{1}\right)$, we have

$$
\begin{aligned}
& \left|u_{n}(t)-u(t)\right| \\
& =\left|f\left(t, x_{n}(t), I^{\alpha-\beta} u_{n}(t), \int_{0}^{t} k(t, s) u_{n}(s) d s\right)-f\left(t, x(t), I^{\alpha-\beta} u(t), \int_{0}^{t} k(t, s) u(s) d s\right)\right| \\
& \leq \psi(t)\left(\left|x_{n}(t)-x(t)\right|+\int_{0}^{t} \frac{(t-s)^{\alpha-\beta-1}}{\Gamma(\alpha-\beta)}\left|u_{n}(s)-u(s)\right| d s+\int_{0}^{t} k(t, s)\left|u_{n}(s)-u(s)\right| d s\right),
\end{aligned}
$$


then

$$
\left\|u_{n}(t)-u(t)\right\| \leq\|\psi\|\left(\left\|x_{n}-x\right\|+\frac{T^{\alpha-\beta}}{\Gamma(\alpha-\beta+1)}\left\|u_{n}-u\right\|+K T\left\|u_{n}-u\right\|\right) .
$$

Thus

$$
\left\|u_{n}-u\right\| \leq \frac{\|\psi\|}{1-\|\psi\|\left(K T+\frac{T^{\alpha-\beta}}{\Gamma(\alpha-\beta+1)}\right)}\left\|x_{n}-x\right\| .
$$

Since $x_{n} \rightarrow x$, then we get $u_{n}(t) \rightarrow u(t)$ as $n \rightarrow \infty$ for each $t \in I$. And let $\varepsilon>0$ be such that, for each $t \in I$, we have $\left|u_{n}(t)\right| \leq \varepsilon$, and $|u(t)| \leq \varepsilon$. Then, we have

$$
\begin{aligned}
|G(t, s)|\left|u_{n}(s)-u(s)\right| & \leq|G(t, s)|\left(\left|u_{n}(s)\right|+|u(s)|\right) \\
& \leq 2 \varepsilon|G(t, s)| .
\end{aligned}
$$

For each $t \in I$, the function $s \rightarrow 2 \varepsilon|G(t ; s)|$ is integrable on $I$. Then applying Lebesgue Dominated Convergence Theorem, then (8) implies that

$$
\left\|A x_{n}-A x\right\| \rightarrow 0 \text { as } n \rightarrow \infty
$$

Consequently, $A$ is continuous.

Step.2: $A$ maps bounded sets into bounded sets in $B_{\rho}$, i.e., $A\left(B_{\rho}\right) \subset B_{\rho}$. Indeed, it is sufficient to demonstrate that there is a positive constant $\rho$ for each $y \in B_{\rho}$, we have $\|A y\| \leq \rho$, show that $A y \in B_{\rho}$.

We have that for each $t \in I$, by the condition $\left(H_{2}\right)$,

$$
|A y(t)|=\left|h(t)+\int_{0}^{T} G(t, s) v(s) d s\right| \leq|h(t)|+\int_{0}^{T}|G(t, s)||v(s)| d s,
$$

where $v(t)=f\left(t, y(t), I^{\alpha-\beta} v(t), \int_{0}^{t} k(t, s) v(s) d s\right)$

$$
\begin{aligned}
|v(t)| & =\left|f\left(t, y(t), I^{\alpha-\beta} v(t), \int_{0}^{t} k(t, s) v(s) d s\right)\right| \\
& \leq F+\psi(t)|y(t)|+\psi(t) \int_{0}^{t} \frac{(t-s)^{\alpha-\beta-1}}{\Gamma(\alpha-\beta)}|v(s)| d s+\psi(t) \int_{0}^{t}|k(t, s)||v(s)| d s .
\end{aligned}
$$

Taking supermum for $t \in I$, we have

$$
\|v\| \leq F+\|\psi\|\|y\|+\|\psi\| \frac{T^{\alpha-\beta}}{\Gamma(\alpha-\beta+1)}\|v\|+\|\psi\| K\|v\| T .
$$

Thus

$$
\|v\| \leq \frac{F+\|\psi\| \varrho}{1-\left(\frac{\|\psi\| T^{\alpha-\beta}}{\Gamma(\alpha-\beta+1)}+\|\psi\| K T\right)}
$$


and

$$
\begin{aligned}
|h(t)| & =\left|y_{\circ}+\frac{\left(y_{T}-y_{\circ}\right) t}{T}\right| \\
& \leq \frac{\left|y_{\circ}\right|(t-T)}{T}+\frac{\left|y_{T}\right| t}{T} \leq\left|y_{T}\right| .
\end{aligned}
$$

Thus (9) implies that, for each $t \in I$,

$$
|A y(t)| \leq\left|y_{T}\right|+\frac{G_{\circ} T(F+\|\psi\| \varrho)}{1-\aleph} \leq \rho .
$$

Taking supermum for $t \in I$, we have

$$
\|A y\| \leq \rho .
$$

Then $A\left(B_{\rho}\right) \subset B_{\rho}$.

Step.3: $A(S)$ is relatively compact.

we prove that $A$ maps bounded sets into equicontinuous sets of $C(I, R)$, i.e, $B \varrho$ is equicontinuous. Now, Let $\forall \epsilon>0, \exists \delta>0$ and $t_{1}, t_{2} \in I, t_{1}<t_{2},\left|t_{2}-t_{1}\right|<\delta$. Then we have

$$
\begin{aligned}
\left|A y\left(t_{2}\right)-A y\left(t_{1}\right)\right| & \leq \int_{0}^{T}\left|G\left(t_{2}, s\right)-G\left(t_{1}, s\right)\right||v(s)| d s \\
& \leq\|v\| \int_{0}^{T}\left|G\left(t_{2}, s\right)-G\left(t_{1}, s\right)\right| d s \\
& \leq \frac{F+\|\psi\| \varrho}{1-\aleph} \int_{0}^{T}\left|G\left(t_{2}, s\right)-G\left(t_{1}, s\right)\right| d s .
\end{aligned}
$$

As $t_{1} \rightarrow t_{2}$, the right-hand side of the above inequality is not dependent on $y$ and tends to zero. Consequently,

$$
\left|A y\left(t_{2}\right)-A y\left(t_{1}\right)\right| \rightarrow 0, \quad \forall\left|t_{2}-t_{1}\right| \rightarrow 0 .
$$

Thus, $\{A y\}$ is equi-continuous on $B_{\varrho}$, and $A$ is compact operator by the Arzela-Ascoli Theorem [6] and in view of the above three steps. Therefore, Operator $A: C(I, R) \rightarrow$ $C(I, R)$ is continuous and completely continuous.

Hence, all the hypotheses of Schauder's fixed point Theorem [6] holds and shows that $A$ has a fixed point on $B_{\varrho}$. Therefore, the IFDP (1)-(2) has a mild solution. The proof is complete.

Our second result is based on Banach's fixed point Theorem to obtain the existence of a unique solution of the IFDP (1)-(2). 
Theorem 2. Let the assumptions of Theorem 1 holds, with

$$
\frac{G_{\circ}\|\psi\| T}{1-\|\psi\|\left(K T+\frac{T^{\alpha-\beta}}{\Gamma(\alpha-\beta+1)}\right)}<1 .
$$

Then the IFDP (1)-(2) has a unique mild solution on I.

Proof. It follows, from Theorem 1, that IFDP (1)-(2) has at least one solution. Therefore, we only need to show that the operator $A$ described in (7) is a contraction.

Now take $x, y \in C(I, R)$. Then for $t \in I$, we have

$$
A x(t)-A y(t)=\int_{0}^{T} G(t, s) u(s) d s-\int_{0}^{T} G(t, s) v(s) d s,
$$

where $u, v \in C(I, R)$ be such that

$$
\begin{aligned}
& u(t)=f\left(t, x(t), I^{\alpha-\beta} u(t), \int_{0}^{t} k(t, s) u(s) d s\right), \\
& v(t)=f\left(t, y(t), I^{\alpha-\beta} v(t), \int_{0}^{t} k(t, s) v(s) d s\right) .
\end{aligned}
$$

Then, for $t \in I$

$$
|A x(t)-A y(t)| \leq \int_{0}^{T} G(t, s)|u(s)-v(s)| d s,
$$

but by condition $\left(H_{2}\right)$, we have

$$
\begin{aligned}
& |u(t)-v(t)| \\
& =\left|f\left(t, x(t), I^{\alpha-\beta} u(t), \int_{0}^{t} k(t, s) u(s) d s\right)-f\left(t, y(t), I^{\alpha-\beta} v(t), \int_{0}^{t} k(t, s) v(s) d s\right)\right| \\
& \leq \psi(t)\left(|x(t)-y(t)|+\int_{0}^{t} \frac{(t-s)^{\alpha-\beta-1}}{\Gamma(\alpha-\beta)}|u(s)-v(s)| d s+\int_{0}^{t} k(t, s)|u(s)-v(s)| d s\right) \\
& \leq\|\psi\|\left(\|x-y\|+\frac{T^{\alpha-\beta}}{\Gamma(\alpha-\beta+1)}\|u-v\|+K\|u-v\| T\right) .
\end{aligned}
$$

Thus

$$
\|u-v\| \leq \frac{\|\psi\|}{1-\|\psi\|\left(K T+\frac{T^{\alpha-\beta}}{\Gamma(\alpha-\beta+1)}\right)}\|x-y\| .
$$

Retrain to (12), we have

$$
|A x(t)-A y(t)| \leq \frac{G_{\circ}\|\psi\| T}{1-\|\psi\|\left(K T+\frac{T^{\alpha-\beta}}{\Gamma(\alpha-\beta+1)}\right)}\|x-y\| .
$$


Taking supermum for $t \in I$, we have

$$
\|A x-A y\| \leq\left(\frac{G_{\circ}\|\psi\| T}{1-\|\psi\|\left(K T+\frac{T^{\alpha-\beta}}{\Gamma(\alpha-\beta+1)}\right)}\right)\|x-y\| .
$$

By $\left(\frac{G_{\circ}\|\psi\| T}{1-\|\psi\|\left(K T+\frac{T^{\alpha-\beta}}{\Gamma(\alpha-\beta+1)}\right)}\right)<1$, the operator $A$ is a contraction. Hence, by Banach's contraction principle, $A$ has a unique fixed point which is a mild solution of the IFDP (1)-(2) on $I$.

Now, we consider the Ulam stability for IFDP (1)-(2). Let $\epsilon>0$ and $\Phi: I \rightarrow R_{+}$be a continuous function. We consider the following inequalities:

$$
\begin{gathered}
\left|{ }^{c} D^{\alpha} y(t)-f\left(t, y(t),{ }^{c} D^{\beta} y(t), \int_{0}^{t} k(t, s)^{c} D^{\alpha} y(s) d s\right)\right| \leq \epsilon(t), t \in I \\
\left|{ }^{c} D^{\alpha} y(t)-f\left(t, y(t),{ }^{c} D^{\beta} y(t), \int_{0}^{t} k(t, s)^{c} D^{\alpha} y(s) d s\right)\right| \leq \Phi(t), t \in I \\
\left|{ }^{c} D^{\alpha} y(t)-f\left(t, y(t),{ }^{c} D^{\beta} y(t), \int_{0}^{t} k(t, s)^{c} D^{\alpha} y(s) d s\right)\right| \leq \epsilon \Phi(t), \quad t \in I .
\end{gathered}
$$

Definition 2. [22] The IFDP (1)-(2) is Ulam-Hyers stable if there exists a real number $c_{f}>0$ such that there exists a solution $x \in C(I, R)$ of $(1)-(2)$ for

$$
|y(t)-x(t)| \leq \epsilon c_{f}, \quad t \in I .
$$

for each solution $y \in C(I, R)$ of the inequality (13).

Definition 3. [22] The IFDP (1)-(2) is generalized to be Ulam-Hyers stable if there is $c_{f} \in C\left(R_{+}, R_{+}\right)$with $c_{f}(0)=0$ so that there is a solution $x \in C(I, R)$ of $(1)-(2)$ with

$$
|y(t)-x(t)| \leq c_{f}(\epsilon), \quad t \in I .
$$

for each $\epsilon>0$ and for each solution $y \in C(I, R)$ of the inequality (13).

Definition 4. [22] The IFDP (1)-(2) is Ulam-Hyers-Rassias stable with respect to $\Phi$ if there exists a real number $c_{f, \Phi}>0$ such that there is a solution $x \in C(I, R)$ of $(1)-(2)$ with

$$
|y(t)-x(t)| \leq \epsilon c_{f, \Phi} \Phi(t), \quad t \in I .
$$

for each $\epsilon>0$ and for each solution $y \in C(I, R)$ of the inequality (15).

Definition 5. [22] The IFDP (2) is generalized Ulam-Hyers-Rassias stable with respect to $\Phi$ if the actual number $c_{f, \Phi}>0$ exists in such a way that for each solution $y \in C(I, R)$ of the inequality (14) there is a solution $x \in C(I, R)$ of (1)-(2) with the solution $x \in C(I, R)$ of the inequality.

$$
|y(t)-x(t)>| \leq c_{f, \Phi} \Phi(t), \quad t \in I .
$$




\subsection{Ulam-Hyers Stability}

Next, we present the following Ulam-Hyers stable result.

Theorem 3. Let the assumptions of Theorem 2 be satisfied. Then IFDP (1)-(2) is Ulam-Hyers stable.

Proof. Let $\epsilon>0$ and let $z \in C(I, R)$ be a function which satisfies the inequality (13),

$$
\left|{ }^{c} D^{\alpha} z(t)-f\left(t, z(t),{ }^{c} D^{\beta} z(t), \int_{0}^{t} k(t, s)^{c} D^{\alpha} z(s) d s\right)\right| \leq \epsilon, \quad t \in I
$$

and let $y \in C(I, R)$ be the unique solution of IFDE (1)-(2) which is by lemma 1 the IFDP (1)-(2) equivalence to fractional order integral equation

$$
y(t)=h(t)+\int_{0}^{T} G(t, s) u(s) d s,
$$

where $u$ is the solution of the functional integral equation

$$
u(t)=f\left(t, h(t)+\int_{0}^{T} G(t, s) u(s) d s, I^{\alpha-\beta} u(t), \int_{0}^{t} k(t, s) u(s) d s\right) .
$$

Operating by $I^{\alpha}$ on both sides of (13), and then integrating, we get

$$
\left|z(t)-h(t)-\int_{0}^{T} G(t, s) v(s) d s\right| \leq \frac{\epsilon T^{\alpha}}{\Gamma(\alpha+1)} .
$$

For each $t \in I$, we have

$$
\begin{aligned}
|z(t)-y(t)| & =\left|z(t)-h(t)-\int_{0}^{T} G(t, s) u(s) d s\right| \\
& \leq\left|z(t)-h(t)-\int_{0}^{T} G(t, s) v(s) d s\right| \\
& +\left|h(t)+\int_{0}^{T} G(t, s) v(s) d s-h(t)-\int_{0}^{T} G(t, s) u(s) d s\right| \\
& \leq \frac{\epsilon T^{\alpha}}{\Gamma(\alpha+1)}+\int_{0}^{T} G(t, s)|v(s)-u(s)| d s \\
& \leq \frac{\epsilon T^{\alpha}}{\Gamma(\alpha+1)}+G_{\circ}\|u-v\| T .
\end{aligned}
$$

Indeed, from proof of Theorem 2, we have

$$
\|u-v\| \leq \frac{\|\psi\|}{1-\|\psi\|\left(K T+\frac{T^{\alpha-\beta}}{\Gamma(\alpha-\beta+1)}\right)}\|z-y\| .
$$


Then, for each $t \in I$

$$
\|z-y\| \leq \frac{\epsilon T^{\alpha}}{\Gamma(\alpha+1)}+\frac{G_{\circ}\|\psi\| T}{1-\|\psi\|\left(K T+\frac{T^{\alpha-\beta}}{\Gamma(\alpha-\beta+1)}\right)}\|z-y\| .
$$

Thus

$$
\|z-y\| \leq \frac{\epsilon T^{\alpha}}{\Gamma(\alpha+1)}\left[1-\left(\frac{G_{\circ}\|\psi\| T}{1-\|\psi\|\left(K T+\frac{T^{\alpha-\beta}}{\Gamma(\alpha-\beta+1)}\right.}\right)\right]^{-1}=\varsigma \epsilon,
$$

for let $\varsigma=\frac{T^{\alpha}}{\Gamma(\alpha+1)}\left[1-\left(\frac{G_{\circ}\|\psi\| T}{1-\|\psi\|\left(K T+\frac{T^{\alpha-\beta}}{\Gamma(\alpha-\beta+1)}\right)}\right]^{-1}\right.$. So, the IFDE (1)-(2) is Ulam-Hyers stable.

By putting $\Phi(\epsilon)=\varsigma \epsilon, \Phi(0)=0$ yields that the IFDE (1)-(2) is generalized Ulam-Hyers stable.

\subsection{Ulam-Hyers-Rassias Stability.}

Now, we state the following Ulam-Hyers-Rassias stable result.

Theorem 4. Assume assumptions $\left(H_{1}\right)-\left(H_{3}\right)$ and

$\left(H_{4}\right)$ The function $\Phi \in C\left(I, R_{+}\right)$is increasing and there exists $\lambda_{\Phi}>0$ such that, for each $t \in J$, we have

$$
I^{\alpha} \Phi(t) \leq \lambda_{\Phi} \Phi(t)
$$

are hold. Then IFDP (1)-(2) is Ulam-Hyers-Rassias stable with respect to $\Phi$.

Proof. Let $z \in C(I, R)$ be a solution of the inequation (15), i.e.,

$$
\left|{ }^{c} D^{\alpha} z(t)-f\left(t, z(t),{ }^{c} D^{\beta} z(t), \int_{0}^{t} k(t, s)^{c} D^{\alpha} z(s) d s\right)\right| \leq \epsilon \Phi, \quad t \in I
$$

and let us assume that $y$ is a solution of the problem (1)-(2). Thus, we have

$$
y(t)=h(t)+\int_{0}^{T} G(t, s) u(s) d s
$$

where $u \in C(I, R)$ such that

$$
u(t)=f\left(t, y(t), I^{\alpha-\beta} u(t), \int_{0}^{t} k(t, s) u(s) d s\right) .
$$


Operating by $I^{\alpha}$ on both sides of the inequality (15) and then integrating, we get

$$
\begin{aligned}
\left|z(t)-h(t)-\int_{0}^{T} G(t, s) v(s) d s\right| & \leq \frac{\epsilon}{\Gamma(\alpha)} \int_{0}^{t}(t-s)^{\alpha-1} \Phi(s) d s \\
& \leq \epsilon \lambda_{\Phi} \Phi(t),
\end{aligned}
$$

where $v \in C(I, R)$ such that

$$
v(t)=f\left(t, z(t), I^{\alpha-\beta} v(t), \int_{0}^{t} k(t, s) v(s) d s\right) .
$$

For each $t \in I$, we have

$$
\begin{aligned}
|z(t)-y(t)| & =\left|z(t)-h(t)-\int_{0}^{T} G(t, s) u(s) d s\right| \\
& \leq\left|z(t)-h(t)-\int_{0}^{T} G(t, s) v(s) d s\right| \\
& +\left|h(t)+\int_{0}^{T} G(t, s) v(s) d s-h(t)-\int_{0}^{T} G(t, s) u(s) d s\right| \\
& \leq \epsilon \lambda_{\Phi} \Phi(t)+\int_{0}^{T} G(t, s)|v(s)-u(s)| d s \\
& \leq \epsilon \lambda_{\Phi} \Phi(t)+\int_{0}^{T} G(t, s)|v(s)-u(s)| d s \\
& \leq \epsilon \lambda_{\Phi} \Phi(t)+G_{\circ}\|v-u\| T .
\end{aligned}
$$

Indeed, from proof of Theorem 2, we have

$$
\|u-v\| \leq \frac{\|\psi\|}{1-\|\psi\|\left(K T+\frac{T^{\alpha-\beta}}{\Gamma(\alpha-\beta+1)}\right)}\|z-y\| .
$$

Then, for each $t \in I$

$$
\|z-y\| \leq \epsilon \lambda_{\Phi} \Phi(t)+\frac{G_{\circ}\|\psi\| T}{1-\|\psi\|\left(K T+\frac{T^{\alpha-\beta}}{\Gamma(\alpha-\beta+1)}\right)}\|z-y\| .
$$

Thus

$$
\|z-y\| \leq\left[1-\frac{G_{\circ}\|\psi\| T}{1-\|\psi\|\left(K T+\frac{T^{\alpha-\beta}}{\Gamma(\alpha-\beta+1)}\right)}\right]^{-1} \epsilon \lambda_{\Phi} \Phi(t)=c_{\Phi} \epsilon \Phi(t),
$$

where

$$
c_{\Phi}=\left[1-\frac{G_{\circ}\|\psi\| T}{1-\|\psi\|\left(K T+\frac{T^{\alpha-\beta}}{\Gamma(\alpha-\beta+1)}\right)}\right]^{-1} \lambda_{\Phi} .
$$

So, the problem IFDP (1)-(2) is Ulam-Hyers-Rassias stable with respect to $\Phi$. 


\section{EXAMPLE}

Example 1. Given the following IFDP:

$$
\begin{gathered}
{ }^{c} D^{\frac{1}{2}}=\frac{2+y(t)+{ }^{c} D^{\frac{4}{3}}+\int_{0}^{1} e^{t-s}{ }^{c} D^{\frac{1}{2}} y(s) d s}{2 e^{t+1}\left(1+y(t)+{ }^{c} D^{\frac{4}{3}}+\int_{0}^{1} e^{t-s c} D^{\frac{1}{2}} y(s) d s\right)}, \quad t \in[0,1] \\
y(0)=1 \quad \text { and } y(1)=1 .
\end{gathered}
$$

Set

$$
f(t, u, v, w)=\frac{2+|u|+|v|+|w|}{2 e^{t+1}(1+|u|+|v|+|w|)} .
$$

Obviously, $f$ is mutually continuous function. In fact, for any $u_{1}, v_{1}, w_{1}, u_{2}, v_{2}, w_{2} \in$ $R$ and $t \in[0,1]$

$$
\left|f(t, u, v, w)-f\left(t, u_{1}, v_{1}, w_{1}\right)\right| \leq \frac{1}{2 e^{2}}\left(\left|u_{1}-u_{2}\right|+\left|v_{1}-v_{2}\right|+\left|w_{1}-w_{2}\right|\right) .
$$

Hence condition $\left(H_{2}\right)$ is satisfied with $\psi(t)=\frac{1}{2 e^{t+1}}$.

Also,

$$
|f(t, u, v, w)|=\frac{1}{2 e^{t+1}}(2+|u|+|v|+|w|), \text { where } F=\frac{1}{e^{2}}, \text { and }\|\psi\|=\frac{1}{2 e^{2}} .
$$

Thus condition

$$
\frac{\|\psi\|}{1-\aleph} \approx 0.393913<1
$$

where $\aleph=\frac{T^{\alpha-\beta}\|\psi\|}{\Gamma(\alpha-\beta+1)}+\|\psi\| K T$, with $T=1, \alpha=\frac{1}{2}, \beta=\frac{4}{3}, F=\frac{1}{e^{2}},\|\psi\|=\frac{1}{2 e}$ and $K=e$. It follows from Theorem 1 that the IFDP (17)-(18) has at least one mild solution on $I$.

Example 2 Consider the following IFDP:

$$
\begin{gathered}
{ }^{c} D^{\frac{3}{2}}=\frac{1}{2 e^{t+1}\left(1+y(t)+{ }^{c} D^{\frac{4}{3}}+\int_{0}^{1} e^{t-s c} D^{\frac{3}{2}} y(s) d s\right)} \\
y(0)=1 \quad \text { and } y(1)=2 .
\end{gathered}
$$

Set

$$
f(t, u, v, w)=\frac{1}{2 e^{t+1}(1+|u|+|v|+|w|)}, \quad t \in[0,1], u, v, w \in R
$$


Clearly, the function $f$ is jointly continuous.

For any $u_{1} \cdot v_{1}, w_{1}, u_{2} \cdot v_{2}, w_{2} \in R$ and $t \in[0,1]$

$$
\begin{aligned}
\mid f\left(t, u_{1}, v_{1}, w_{1}\right) & -f\left(t, u_{2}, v_{2}, w_{2}\right) \mid \\
& \leq\left|\frac{1}{2 e^{t+1}}\left[\frac{1}{\left(1+\left|u_{1}\right|+\left|v_{1}\right|+\left|w_{1}\right|\right)}-\frac{1}{\left(1+\left|u_{2}\right|+\left|v_{2}\right|+\left|w_{2}\right|\right)}\right]\right| \\
& \leq\left|\frac{1}{2 e^{t+1}}\left[\frac{\left|u_{1}-u_{2}\right|+\left|v_{1}-v_{2}\right|+\left|w_{1}-w_{2}\right|}{\left(1+\left|u_{1}\right|+\left|v_{1}\right|+\left|w_{1}\right|\right)\left(1+\left|u_{2}\right|+\left|v_{2}\right|+\left|w_{2}\right|\right)}\right]\right| \\
& \leq \frac{1}{2 e^{2}}\left(\left|u_{1}-u_{2}\right|+\left|v_{1}-v_{2}\right|+\left|w_{1}-w_{2}\right|\right) .
\end{aligned}
$$

Hence condition $\left(H_{2}\right)$ is hold with $\|\psi\|=\frac{1}{2 e^{2}}<1$. From (5) the function $G$ is given by

$$
G(t, s)=\left\{\begin{array}{lc}
\frac{(t-s)^{\frac{1}{2}}}{\Gamma(\alpha)}-\frac{t(1-s)^{\frac{1}{2}}}{T \Gamma(\alpha)} & 0 \leq s \leq t \leq 1, \\
\frac{-t(1-s)^{\frac{1}{2}}}{T \Gamma(\alpha)} & 0 \leq t \leq s \leq 1,
\end{array}\right.
$$

Clearly $G_{0}<2$. Thus We shall check that condition 10 thus

$$
\frac{G_{\circ}\|\psi\| T}{1-\|\psi\|\left(K T+\frac{T^{\alpha-\beta}}{\Gamma(\alpha-\beta+1)}\right)}=0.1821179198<1 .
$$

is hold with $T=1, \alpha=\frac{3}{2}, \beta=\frac{4}{3},\|\psi\|=\frac{1}{2 e^{2}}$ and $K=e$. It follows from Theorem 2 that the problem (19)-(20) as a unique solution on $I$.

\section{CONCLUSION}

In this current research paper. First, the equivalence between IFDP (1)-(2) and the Volterra integration equation (3) was developed in our study. Secondly, the existence and uniqueness of mild solutions for boundary value problems of implicit fractional order differential equations were established based on Schauder's fixed point theorem, and Banach contraction principle. We found the Ulam Hayers stability and the generalized Ulam Hayers stability, the stability of Ulam - Hyers - Rassias and the generalized stability of Ulam - Hyers - Rassias allowed on the implicit differential equation of fractional order, supplemented with fractional integral type boundary conditions. Finally, we end the article with illustrations examples to prove the applicability of the result obtained. 


\section{REFERENCES}

[1] E. Ahmed, A.M.A. El-Sayed, A.E.M. El-Mesiry and H. A. A. El-Saka, Numerical solutions for the fractional replicator equation, International Journal of Modern Physics C, 16(7) (2005), 1-9.

[2] S. Abbas and M. Benchohra, On the generalized Ulam-Hyers-Rassias stability for Darboux problem for partial fractional implicit differential equations, Appl. Math. E-Notes, 14 (2014), 20-28.

[3] R.P Agarwal, M. Benchohra and S. Hamani, Boundary value problems for frac- tional differential equations, Georgian Mathematical Journal, 16(3) (2009), 401-411.

[4] M. Benchohra, J.R. Graef and S. Hamani, Existence results for boundary value problems with nonlinear fractional differential equations, Applicable Analysis, 87(7)(2008), 851-863. Zbl 1198.26008.

[5] M. Benchohra, S. Hamani and S.K. Ntouyas, Boundary value problems for differential equations with fractional order, Surveys in Mathematics and its Applications, 3 (2008), 1-12.

[6] R. F. Curtain and A. J. Pritchard, Functional analysis in modern applied mathematics, Academic press. (1977).

[7] A.M.A. El-Sayed, Nonlinear functional differential equations of arbitrary orders, Nonlinear Analysis: Theory, Methods And Applications, 33(2) (1998), 181-186.

[8] A.M.A. El-Sayed, A. Elsonbaty, A.A. Elsadany and A.E. Matouk, Dynamical Analysis and Circuit Simulation of a New Fractional-Order Hyperchaotic System and Its Discretization, Int. J. Bifurcat. Chaos, 26(13) (2016), 1-35.

[9] A.M.A. El-Sayed and Sh.M Al-Issa, Existence of integrable solutions for integro-differential inclusions of fractional order; coupled system approach, Journal of Nonlinear Sciences and Applications, 13 (2020), 180-186.

[10] A.M.A. El-Sayed and Sh. M Al-Issa, On a set-valued functional integral equation of Volterra-Stiltjes type, J. Math. Computer Sci., 21(4) (2020), 273-285.

[11] Sh.M Al-Issa and N.M. Mawed. Results on solvability of nonlinear quadratic integral equations of fractional orders in Banach algebra. J.Nonlinear Sci. Appl., 14(4) (2021), 181-195.

[12] A.M.A. El-Sayed, Sh.M Al-Issa and M.H. Hijazi, Existence results to a class of first-order functional integro-differential inclusions, Matrix Science Mathematic, 4(2) (2020), 44-50. 
[13] A.M.A. El-Sayed, A.E.M. El-Mesiry and H.A.A. El-Saka, Numerical solution for multi-term fractional (arbitrary) orders differential equations, Comput. Appl. Math., 23(1) (2004), 33-54.

[14] A.M.A. El-Sayed and F.M. Gaafar, Fractional order differential equations with memory and fractional-order relaxation-oscillation model, Pure Math. Appl., 12(3) (2001), 296-310.

[15] A.M.A. El-Sayed, F.M. Gaafar and H.H. Hashem, On the maximal and minimal solutions of arbitrary orders nonlinear functional integral and differential equations, Math. Sci. Res. J., 8(11) (2004), 336-348. MR2572663(2010j:34003). Zbl 1179.26011.

[16] A.R. Elsonbaty and A.M.A. El-Sayed Further nonlinear dynamical analysis of simple jerk system with multiple attractors Nonlinear Dyn, 87(2) (2017), 1169-1186.

[17] S. Jung and K. Lee, Hyers-Ulam stability of first order linear partial differential equations with constant coefficients, Math. Inequal. Appl., 10(2) (2007), 261-266.

[18] A.A. Kilbas, H.M. Srivastava and J.J. Trujillo, Theory and Applica- tions of Fractional Differential Equations, Theory and applications of fractional differential equations. elsevier, 204 (2006). MR2218073(2007a:34002).Zbl 1092.45003.

[19] V. Lakshmikantham, S. Leela and J. Vasundhara, Stability theory for set differential equations, Dynamics of Continuous, Discrete and Impulsive Systems Series A, 11 (2004), 181-189.

[20] M.A.E. Herzallah, A. M.A. El-Sayed and D. Baleanu, On the fractional-order diffusion-wave process Rom. Journ. Phys., 55(3-4) ( 2010), 274-284.

[21] I. Podlubny and A.M.A. EL-Sayed, On two defintions of fractional calculus, Preprint UEF, (1996), 3-69.

[22] I.A. Rus, Ulam stability of ordinary differential equations, Studia Universitatis Babes-Bolyai, Mathematica, 4 (2009), 125-133. 
